Physics, Chemistry, and Dynamics of Interplanetary Dust

ASP Conference Series, Vol. 104, 1996

Bo A. S. Gustafson and Martha S. Hanner (eds.)

\title{
Modelling the Ejection of Meteoroids from Comets
}

\author{
J. Jones and P. Brown, \\ Physics Department, University of Western Ontario, London, Ontario, \\ Canada, N6A $3 K^{\gamma}$
}

\begin{abstract}
We have reworked Whipple's (1951) theory of the ejection of meteoroids from comets to include the effects of cooling by the sublimation of the cometary ice and the adiabatic expansion of the escaping gases. We consider only those particles moving significantly slower than the gas speed and find that the inclusion of these effects does not yield results much different from Whipple's theory. We have extended the theory to include the case of an active area in the form of a spherical cap and have shown how the characteristics of the ejection process change when the cap is in the form of a pit or a depression. We present a empirical formulae which shculd be useful to modellers of meteor stream evolution.
\end{abstract}

\section{Introduction}

Whipple's theory of the ejection of dust particles is widely used by the meteor scientific community in meteor stream evolution studies to obtain the initial distribution of orbits of the meteoroids after they are ejected from the comet. While encouraging agreement between prediction and observation has been obtained in many cases, the time is ripe to try to examine, and if possible, improve our basic model.

In Whipple's model, the comet was considered to be a sphere of ice in which are embedded stony particles. The temperature of the cometary surface was supposed to be determined by the balance of incident solar radiation and blackbody radiation from the surface. The evaporated water molecules were taken to leave the surface radially with a speed equal to the mean speed of molecules of an ideal gas at the surface temperature. The motion of the stony particles, after release from the ice on sublimation, was calculated assuming that their velocity was always small compared with the that of the gas. Finally it was assumed that evaporation was important only on the sunlit face of the comet. Although, since 1951, Whipple's theory has been considerably improved upon (see for example Probstein, 1968; Gombosi et al 1986), most of these efforts have been directed towards the behavior of the small dust particles responsible for much of the light reflected from comet tails and which are typically of radius $10 \mu \mathrm{m}$ or less. For comparison, a $0^{M}$ meteoroid travelling at $30 \mathrm{~km} / \mathrm{s}$ has a radius of about 3 $\mathrm{mm}$. In this paper we are interested in the motion of meteoroid-size particles for which many of the effects which dominate the motions of the micron sized dust particles are not important. Rather than try to adapt the above mentioned small-particle ejection schemes to our purpose, we have sought to remedy some 
of the shortcomings of Whipple's theory by including the effects of cooling by the sublimation of the ice and the adiabatic expansion of the escaping gases as was done by Probstein (1968) for the fine dust particles.

\section{The flow of the gas away from the comet.}

For $r<2.5 A U$ sublimation cooling is the primary mechanism of heat loss from the cometary surface. We have found that a convenient approximation for $T s(r)$ in this region is

$$
T_{s}=204.5 r^{-0.0761}(K) .
$$

With a Maxwellian distribution of speeds the mean component, $v_{s}$, of an escaping gas molecule perpendicular to the surface is given by

$$
v_{s}=\sqrt{\pi k T_{s} / 2 \mu}
$$

where $k$ is Boltzmann's constant and $\mu$ the molecular mass.

As the gas recedes from the comet it expands as well as accelerates due to the pressure gradient. Combining the Navier-Stokes equation with the adiabatic gas law and integrating we find

$$
v^{2}=v_{s}^{2}+v_{s}^{2} \frac{4 \gamma}{\pi(\gamma-1)}\left[1-\left(\frac{\rho}{\rho_{s}}\right)^{\gamma-1}\right]
$$

where the subscripts denote surface values.

At this point it is convenient to work in terms of normalized variables defined by the relations $\rho_{n}=\rho / \rho_{s}$ and $v_{n}=v / v_{s}$. For water vapor $\gamma=1.31$ and hence we find

$$
v_{n}=\sqrt{1+5.38\left(1-\rho_{n}^{\gamma-1}\right)} .
$$

The normalized mass flux, $\mathbf{J}_{\mathbf{n}}$, is given by $\mathbf{J}_{\mathbf{n}}=\rho \mathbf{v}_{\mathbf{n}}$ which allows us to calculate $\rho_{n}$ given $\mathbf{J}_{\mathbf{n}}$ and hence $\mathbf{v}_{\mathbf{n}}$.

\section{Active area in form of a spherical cap}

Because we expect the flow to be irrotational and the viscosity to be negligible we can express the gas flux in terms of a scalar potential so that the continuity equation reduces to $\nabla \cdot \mathbf{J}=\mathbf{0}$ which allows us to define $\mathbf{J}$ in terms of a scalar potential $\Phi$ which can be obtained by solving Laplace's equation subject to the boundary condition that the flux is radially uniform over the cap and zero elsewhere. For an active region in the form of a spherical cap of semi-angle $\alpha$, $\Phi$, is given by

$$
\phi=\sum_{0}^{\infty} \frac{1}{2(l+1)}\left[P_{l+1} \cos (\alpha)+P_{l-1} \cos (\alpha)\right] R^{-(l+1)} P_{l} \cos (\theta)
$$

where $\theta$ is the usual polar angle and $R$ is the distance from the comet in units of the cometary radius. Thus $\Phi$ and $\mathrm{J}_{\mathrm{n}}$ and hence $v_{n}$ can be calculated at any point outside the comet. 
The equation of motion of particles that have final speeds small compared with the final neutral gas speed is

$$
\frac{d \mathbf{V}}{\mathrm{dt}}=\frac{A_{p} D}{2 m} \rho v^{2} \frac{\mathbf{J}}{|\mathbf{J}|}
$$

where $A_{p}$ is the shape factor and $\mathrm{D}$ the drag coefficient of the meteoroid.

Using equation 6 , we have integrated the trajectories of spherical dust particles in the velocity field determined via equation 5 out to $50 R_{c}$ assuming them to be initially at rest as they become detached from the cometary surface and that $D=2.89$ (Whipple,1951).

\section{Results of calculations}

We found the final speed of the particles to vary with cap angle much as expected: small active areas should behave more or less like small comets and produce relatively low final speeds. We have fitted an empirical formula to our data that is suitable for use with numerical models:

$$
v_{\infty}=41.7[\sin (\alpha / 2)]^{0.37}[\cos (\chi)]^{0.519} R_{c}^{1 / 2} m^{-1 / 6} \rho^{-1 / 3} r^{-1.038}
$$

where $\chi$ is the local solar zenith angle. For a cap angle of $10^{\circ}$ we find that $V_{\infty}=12.8(\mathrm{~m} / \mathrm{s})$ for a $10^{-3} \mathrm{~kg}$ particle of density $800 \mathrm{~kg} \mathrm{~m}^{-3}$ ejected at $1 \mathrm{AU}$ from the Sun with $R_{c}=5 \mathrm{~km}$ and $\chi=0$.

The water vapor, as it recedes from the comet, expands laterally and the lateral expansion of the gas close to the comet broadens the initially well-defined dust jet so that it becomes a "dust cone". For example, the dust released from a cap of semi-angle $10^{\circ}$ ends up being distributed of a cone of semi-angle about $43.4^{\circ}$, while dust from a $60^{\circ}$ cap is broadened into a final ejection cone of semiangle of about $90^{\circ}$. We have found the following empirical formula describes the variation of $\phi_{\infty}$, the half-width of this "exit cone", with $\alpha$ :

$$
\phi_{\infty}=33.7+0.9958 \alpha+0.002803 \alpha^{2}-2.123 \times 10^{-5} \alpha^{3}
$$

Thus for $\alpha=60^{\circ}$ which corresponds to a hemispherical exit cone far from the comet, we find

$$
V_{\infty}=32.3 R_{c}^{1 / 2} m^{-1 / 6} \rho^{-1 / 3} r^{-1.038} \mathrm{~m} / \mathrm{s}
$$

which is to be compared with Whipple's formula

$$
V_{\infty}=25.4 R_{c}^{1 / 2} m^{-1 / 6} \rho^{-1 / 3} r^{-1.125} \mathrm{~m} / \mathrm{s} .
$$

The final speed of the meteoroid evidently does not depend critically on the details of the gas velocity field.

\section{The effect of depressed active areas.}

The observations of the narrow jets of gas and dust from Comet $\mathrm{P} /$ Halley indicate that the active areas are in the form of pits in the cometary crust. The 
effect of the pit is to prevent lateral expansion of the gas so that its velocity inside the pit is constant and the dust grains released from the floor of the pit are subject to a short period of relatively high acceleration before they emerge at the surface of the comet. Proceeding as before, for a pit whose dimensions are large compared with the mean-free-path of the gas, we incorporate the effect of the pit of depth $\delta$ by giving the dust particles at the surface a small initial velocity of magnitude, $V_{s}$, given by

$$
V_{s}=v_{s} \sqrt{A_{p} D \rho_{s} \delta / m} .
$$

Of particular interest is the behavior of dust ejected from a pit on a plane surface since this is the limiting case for small cap angles. Calculating as before and extrapolating to the case of very small cap angles, we found the following useful empirical formulae for the planar case for a pit of diameter $\Delta$ :

$$
\phi_{\infty}(\delta / \Delta)=\sqrt{\frac{1256-2.08(\delta / \Delta)}{1+14.69(\delta / \Delta)}}\left(^{\circ}\right)
$$

and

$$
V_{\infty}(\delta / \Delta) / V_{\infty}(0)=\sqrt{1+0.585(\delta / \Delta)} .
$$

\section{Conclusions}

The final speeds of meteoroids that are substantially less than that of the escaping gas do not depend critically on either the cooling mechanism or whether the escaping gases expand isothermally or adiabatically so that Whipple's original model works fairly well for hemispherical ejection. This implies that the coupling between the motion of the meteoroid-sized particles and the gas is greatest close to the surface of the comet and that the motion of the gas far from the comet has little influence in this size regime. We have investigated the effect of sublimation from an active area in the form of a circular cap and find that even a very small active region results in dust particles being ejected in a cone of semi-angle of close to $34^{\circ}$. We have also shown how the dust particles are ejected if the active area is in the form of a pit or depression. To make this paper useful to modellers of meteoroid stream evolution we present empirical formulae which summarize the results of our numerical calculations.

Acknowledgments. This work was supported by a grant from the Natural Sciences and Engineering Research Council of Canada. The authors wish to thank Dr. W Jones, Dr. D. Steel and Dr. D.W. Hughes for valuable criticism and suggestions.

\section{References}

Gombośsi, T.I., Nagy, A.F. \& Cravens, T.E. 1986 Rev. Geophys. 24, 667.

Probstein, R.F.,1968 in "Problems of Hydrodynamics and Continuum Mechanics", p 568. Society for Industrial and Applied Mathematics, Philadelphia, Pa.

Whipple, F.L. 1951. ApJ, 113, 464. 